\title{
BMJ Open Comparative safety of the sodium glucose co-transporter 2 (SGLT2) inhibitors: a systematic review and meta-analysis
}

Jennifer R Donnan, ${ }^{1}$ Catherine A Grandy, ${ }^{1}$ Eugene Chibrikov, ${ }^{1}$ Carlo A Marra, ${ }^{1,2}$ Kris Aubrey-Bassler, ${ }^{3}$ Karissa Johnston, ${ }^{1}$ Michelle Swab, ${ }^{3}$ Jenna Hache, ${ }^{1}$ Daniel Curnew, ${ }^{1}$ Hai Nguyen, ${ }^{1}$ John-Michael Gamble ${ }^{1,4}$

To cite: Donnan JR, Grandy CA, Chibrikov E, et al. Comparative safety of the sodium glucose co-transporter 2 (SGLT2) inhibitors: a systematic review and meta-analysis. BMJ Open 2019;9:e022577. doi:10.1136/ bmjopen-2018-022577

- Prepublication history and additional material for this paper are available online. To view these files, please visit the journal online (http://dx.doi. org/10.1136/bmjopen-2018022577).

Received 2 March 2018 Revised 14 June 2018 Accepted 2 November 2018

Check for updates

(C) Author(s) (or their employer(s)) 2019. Re-use permitted under CC BY-NC. No commercial re-use. See rights and permissions. Published by BMJ.

${ }^{1}$ School of Pharmacy, Memorial University of Newfoundland,

St. John's, Newfoundland and Labrador, Canada

${ }^{2}$ School of Pharmacy, University of Otago, Dunedin, New Zealand ${ }^{3}$ Faculty of Medicine, Memorial University, St. John's,

Newfoundland and Labrador, Canada

${ }^{4}$ School of Pharmacy, Faculty of Science, University of Waterloo, Waterloo, Ontario, Canada

Correspondence to

Dr John-Michael Gamble;

jm.gamble@uwaterloo.ca

\section{ABSTRACT}

Objective To estimate the association between the use of sodium glucose co-transporter-2 (SGLT2) inhibitors and postmarket harms as identified by drug regulatory agencies.

Design We conducted a systematic review and metaanalysis of randomised controlled trials (RCT). Six large databases were searched from inception to May 2018. Random effects models were used to estimate pooled relative risks (RRs).

Intervention SGLT2 inhibitors, compared with placebo or active comparators.

Primary outcomes Acute kidney injury (AKI), diabetic ketoacidosis (DKA), urinary tract infections (UTI), bone fractures and lower limb amputations.

Results We screened 2418 citations of which 109 were included. Most studies included one of four SGLT2 inhibitors, dapagliflozin, canagliflozin, empagliflozin and ipragliflozin. When compared with placebo, SGLT2 inhibitors were found to be significantly protective against $\mathrm{AKI}(\mathrm{RR}=0.59 ; 95 \% \mathrm{Cl} 0.39$ to $0.89 ; l^{2}=0.0 \%$ ), while no difference was found for DKA (RR $0.66 ; 95 \% \mathrm{Cl} 0.30$ to $1.45, \mathrm{I}^{2}=0.0 \%$ ), UTI (RR $1.02 ; 95 \% \mathrm{Cl}$ 0.95 to $1.09, l^{2}=0.0 \%$ ) or bone fracture (RR $0.87 ; 95 \% \mathrm{Cl}$ 0.69 to $\left.1.09, I^{2}=1.3 \%\right)$. Three studies reported on amputation, with one finding a significant increase risk. No increased risk for either outcome was found when compared with active controls. Subgroup analysis did show an increased risk of UTI with dapagliflozin only (RR $1.21 ; 95 \% \mathrm{Cl} 1.02$ to 1.43 , $\mathrm{I}^{2}=0.0 \%$ ), but no other analysis supported an increased risk of AKI, DKA, UTI or fracture.

Conclusions Current evidence from RCTs does not suggest an increased risk of harm with SGLT2 inhibitors as a class over placebo or active comparators with respect to AKI, DKA, UTI or fracture. However, wide Cls for many comparisons suggest limited precision, and therefore clinically important adverse events cannot be ruled out. Dapagliflozin, appears to independently increase the risk of UTI, although the mechanism for this intraclass variation in risk is unclear. PROSPERO registration number CRD42016038715.

\section{INTRODUCTION}

The sodium glucose co-transporter 2 (SGLT2) inhibitors are novel glucose-lowering therapies
Strengths and limitations of this study

- This study provides a comprehensive systematic review of potential serious adverse events related to use of sodium glucose co-transporter-2 (SGLT2) inhibitors identified by drug regulatory agencies.

- This study considered select outcomes to provide focused attention on the issues concerning regulators; however, this means that additional knowledge of the clinical benefits and harms needs to be considered before applying the results of this study.

- Several of the outcomes (eg, acute kidney injury, diabetic ketoacidosis, limb amputations) we evaluated occur infrequently and, in some cases, were not reported by individual studies.

- Certain outcomes may have been inadequately characterised within study reports. For example, while urinary tract infections were commonly reported among randomised controlled trials included in this meta-analysis, data on complicated versus uncomplicated infections were not.

- Our objective is to summarise the current state of knowledge surrounding key postmarket safety concerns of the SGLT2 inhibitors compared with active and non-active comparators in patients with type 2 diabetes.

available for the management of type 2 diabetes. Clinical guidelines recommend the SGLT2 inhibitors as one of numerous potential pharmacological approaches for secondline therapy following metformin failure or intolerance. ${ }^{12}$ Some clinical guidelines recommend the SGLT2 inhibitors, empagliflozin or canagliflozin, or the glucagon-like peptide-1 (GLP-1) receptor agonist, liraglutide, as preferred second-line therapies in patients with cardiovascular disease who have failed to achieve glycaemic control while on monotherapy. ${ }^{1}$ This paradigm shift in the management of type 2 diabetes is largely supported by evidence from recent landmark 
clinical trials. $^{3-6}$ In 2015, the Empagliflozin Outcome Event Trial in Type 2 Diabetes Mellitus Patients-Removing Excess Glucose (EMPA-REG OUTCOME) trial showed that the SGLT2 inhibitor, empagliflozin, significantly reduced the risk for composite end point of cardiovascular death, myocardial infarction or stroke by $14 \%$ and all-cause mortality by $32 \%$, in a population with existing cardiovascular disease. ${ }^{5}$ The Canagliflozin Cardiovascular Assessment Study (CANVAS), Liraglutide Effect and Action in Diabetes: Evaluation of Cardiovascular Outcome Results (LEADER) trial and the Trial to Evaluate Cardiovascular and Other Long-term Outcomes with Semaglutide in Subjects with Type 2 Diabetes (SUSTAIN-6) have also demonstrated similar benefits with canagliflozin, liraglutide and semaglutide respectively. ${ }^{34}$

Considering the relative potential harms and benefits, clinicians and policy makers must continue to integrate new pharmacotherapeutic evidence to optimise health outcomes. Although the EMPA-REG trial showed that the SGLT2 inhibitor, empagliflozin, significantly reduces the risk of cardiovascular morbidity and mortality, regulatory agencies including the Food and Drug Administration (FDA), the European Medicines Associations and Health Canada have issued safety warnings for several adverse events. These include acute kidney injury (AKI), diabetic ketoacidosis (DKA), urinary tract infections (UTI), bone fractures and lower limb amputations, based primarily on case report data. ${ }^{7-14}$

With respect to AKI, there is conflicting information coming forward from clinical trials and case reports. Despite early indication of a protective effect from SGLT2 inhibitors, ${ }^{15}$ the FDA published in a safety communication in June 2016 that 101 cases of AKI were reported among users of canagliflozin and dapagliflozin. ${ }^{12}$ In May 2015, the FDA published a safety update indicating an increased risk of UTI and DKA. Among patients taking SGLT2 inhibitors, they identified 19 cases of life-threatening infections that originated as a UTI, and 73 cases of DKA. However, to date clinical trial evidence does not support these potential risks. Previously published meta-analyses of randomised controlled trials (RCT) found no increased risk of UTIs, except within a subgroup of dapagliflozin, ${ }^{15-18}$ and one study found an increased risk with empagliflozin $25 \mathrm{mg}$ users. ${ }^{18}$ A previous meta-analysis on the risk of DKA currently showed no increased risk. ${ }^{19}$ In January 2016, the FDA issued an expanded warning regarding a potential increased risk for fracture with canagliflozin. ${ }^{9}$ Two published meta-analyses ${ }^{20} 21$ of SGLT2 inhibitors did not find an increased risk, nor did a pooled analysis of eight canagliflozin trials. ${ }^{22}$ Finally, in May 2017, the FDA supported earlier speculation of increased risk of low limb amputation ${ }^{11}$ with evidence gathered from re-analysis of the CANVAS and CANVAS renal end points (CANVAS-R) trials, demonstrating a twofold increased risk. ${ }^{23}$ No meta-analysis of RCTs currently exists with respect to amputation.

In light of recent guideline changes that promote preferential use of the SGLT2 inhibitors, clinicians and policy makers need to continue examining the potential risks to their patients. Our objective is to address the current knowledge gap surrounding the postmarket safety of the SGLT2 inhibitors compared with active and non-active comparators in patients with type 2 diabetes. We have conducted a systematic review and meta-analysis of RCTs to estimate the risk of AKI, DKA, UTI, bone fracture and lower limb amputation.

\section{METHODS AND ANALYSIS \\ Study design}

This study has been designed in accordance with the Preferred Reporting Items for Systematic Reviews and Meta-Analyses statement on systematic reviews and meta-analysis. ${ }^{24}$ This protocol has been registered (CRD42016038715) with PROSPERO (International Prospective Register of Systematic Reviews). ${ }^{25} 26$

\section{Patient involvement}

Patients were not engaged in the development of this protocol.

\section{Search strategy}

A comprehensive search strategy was developed with an experienced health science librarian (MS). The search strategy for published studies was developed in the PubMed database, and comprised keywords and MEDLINE controlled vocabulary or medical subject headings. A methodological search filter was applied to identify $\mathrm{RCTs}^{26}$ and the search was limited to English language publications. This search strategy served as a template for additional search strategies tailored to other databases, including the Cochrane Library, EMBASE and International Pharmaceutical Abstracts. In addition, the reference lists of topical review articles, editorials and included studies were hand-searched to identify other potentially relevant studies. A list of search terms is provided in section 1 of the online supplementary appendix.

The search for unpublished studies and materials included ProQuest Dissertations and Theses Global (ProQuest), and clinical trial registries (ClinicalTrials. gov). Inclusion of unpublished data from the FDA has been shown to substantially impact the effect estimates of meta-analyses of drug trials. ${ }^{27}$

\section{Eligibility criteria}

We included RCTs with a study population consisting of patients 18 years of age and older with a diagnosis of type 2 diabetes. Studies were required to have a formal definition of type 2 diabetes based on established diagnostic criteria during the time of the study. No restriction was applied with respect to history of diabetes medication use. One of the RCT study groups was required to be one of the following SGLT2 inhibitors: canagliflozin, dapagliflozin, empagliflozin, ipragliflozin or any other investigational or approved SGLT2 inhibitor during study period. Eligible comparators included second-generation 
sulfonylureas (glyburide, gliclazide, glimepiride, glipizide-first-generation sulfonylureas excluded as they have a limited role in clinical practice), basal insulins (Neutral Protamine Hagedorn (NPH), lente, glargine, detemir, degludec), dipeptidyl peptidase-4 (DPP-4) inhibitors (alogliptin, linagliptin, saxagliptin, sitagliptin), GLP-1 receptor agonists (dulaglutide, exenatide, liraglutide), thiazolidinediones (pioglitazone, rosiglitazone), $\alpha$-glucosidase inhibitors (acarbose) or placebo/no treatment. All premixed or acute care insulin protocols were excluded. Any investigational agents other than SGLT2 inhibitors were excluded.

The outcomes of this study include the serious safety events as highlighted through the federal regulatory drug safety communications. ${ }^{7-11}$ These include AKI, DKA, UTI, bone fractures and lower limb amputations.

Studies were eligible regardless of duration of follow-up, or publication date; however, non-English citations were excluded. Language restriction does not appear to bias estimates of therapeutic interventions. ${ }^{28} 29$

\section{Study selection and data extraction}

We used DistillerSR, a systematic review software ${ }^{30}$ for screening and data extraction. Studies went through a two-level screening process. First, titles and abstracts were reviewed using the inclusion and exclusion criteria. Studies that met those criteria, or where a clear decision could not be made, were moved to second-level screening. At level two screening, full-text articles were retrieved and the same criteria applied. Duplicate screening was carried out using the 'liberal accelerated' method at both level one and level two, which was first applied by Khangura $e t ~ a l .{ }^{31}$ This method involves having a second reviewer only evaluate studies that were deemed not relevant by the lead reviewer. This reduces the overall number of papers that require duplicate screening without increasing the risk of having appropriate studies inadvertently excluded.
Information extracted included study characteristics (country, definitions of exposure(s) and controls), patient characteristics (sex, age, duration of diabetes) and outcome data (a complete list of extracted variables is available in section 2 of the online supplementary appendix). Where the data conflicted between the published paper and other sources (eg, ClinicalTrials. gov), the data from the published paper were used. Data were only supplemented from other sources when gaps in information existed. In cases where more than one publication reported data on the same study, preference was taken to studies that reported numbers of events (vs only relative risk (RR) or HR) and the most recent were used for data extraction. The exception to this rule was when there was a change to the intervention or comparator groups (eg, drug, dose, etc) for study extensions, then data from the original publication were used. Any disagreements were resolved through discussion and consensus. Where necessary, a third reviewer was consulted. All DistillerSR screening and extraction forms were created a priori and piloted using a small sample of eligible studies.

\section{Risk of bias assessment}

Each included study was critically appraised using the Cochrane Collaboration domain-based tool for assessing the risk of bias for RCTs. ${ }^{32}$ This tool captures six main sources of bias, including: randomisation sequence, allocation concealment, blinding of participant and researcher, blinded outcome assessment, incomplete outcome data and selective reporting. A seventh category captures any other potential sources of bias. Bias was assessed at the study level. Low risk of bias was defined as an assessment on the risk of bias tool that included no more than two categories with 'unclear risk'. Studies were defined as high risk if they had: three or more categories of 'unclear risk'; one or more categories of 'medium risk'

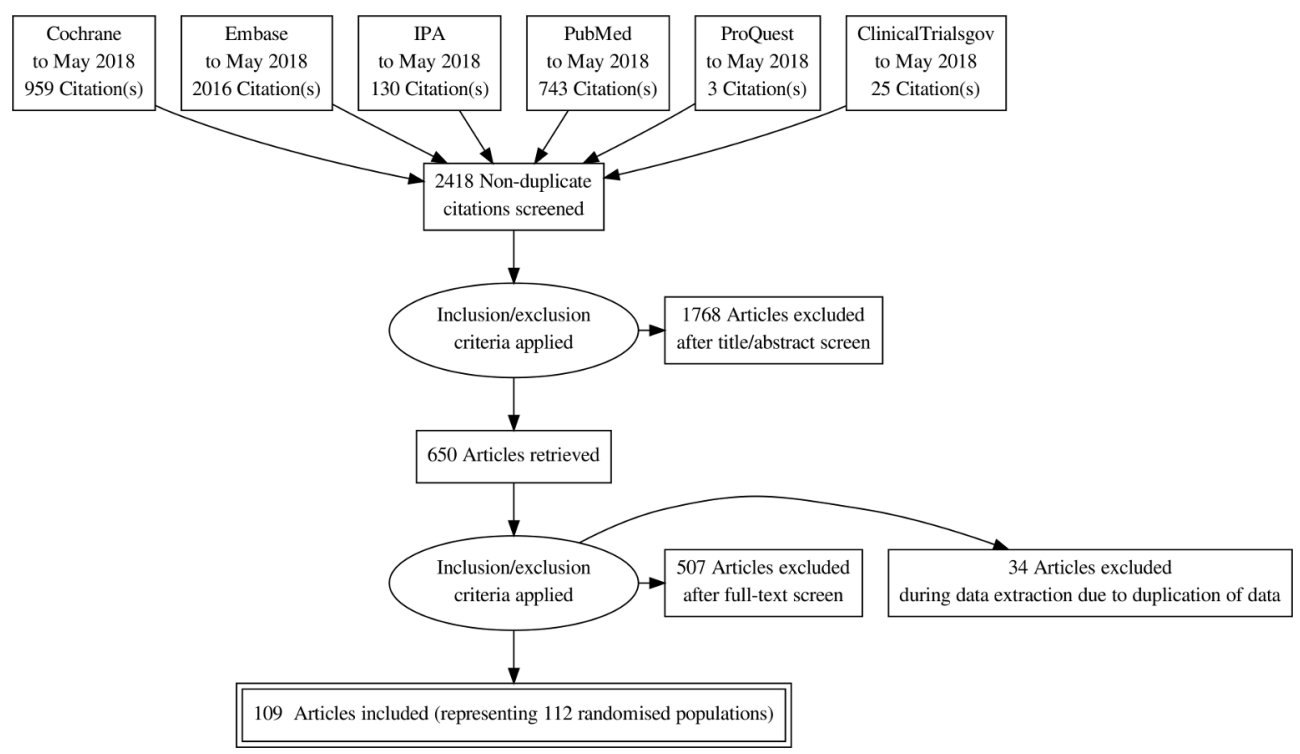

Figure 1 Flow diagram for included studies. 


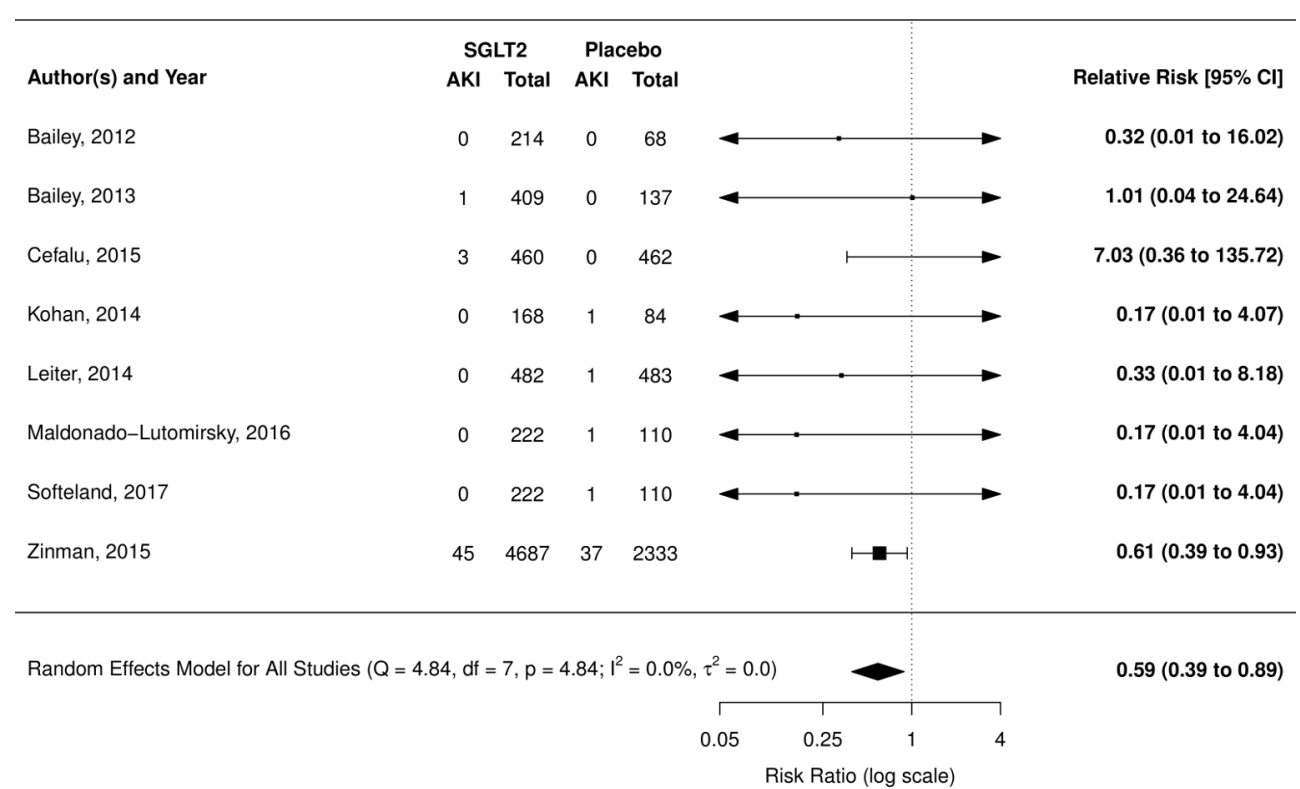

Figure 2 Risk of acute kidney injury (AKI) with sodium glucose co-transporter-2 (SGLT2) inhibitors compared with placebo.

or one or more categories of 'high risk'. Publication bias was examined using funnel plots.

\section{Data synthesis}

We conducted a series of pair-wise random effects meta-analyses to estimate the pooled treatment effect using RRs, using the restricted maximum likelihood method. ${ }^{33}$ The primary analysis was split into two comparisons, with the first between SGLT2 inhibitors and placebo, and the second between SGLT2 inhibitors and any active comparator. If there were zero events reported, a default value of 0.5 was added to all groups within that study. Statistical heterogeneity was evaluated using the $\mathrm{I}^{2}$ statistic, with significant heterogeneity defined as an $\mathrm{I}^{2}>50 \% .^{34}$ To explore treatment effect heterogeneity, we conducted numerous subgroup analyses according to individual SGLT2 inhibitors, risk of bias and concurrent use of other diabetes medications. Concurrent/prior use was defined as any previous use of antidiabetic agents that were used prior to enrolment or added as background therapy after enrolment. If patients could be therapy-naive or have used other medications to meet enrolment criteria, then they were categorised as concurrent/prior use. Treatment-naïve was defined as patients who have never had an antidiabetic medication in the past, have not been on any other antidiabetic medication in weeks leading up to enrolment or were able to go through a washout prior to enrolment. We also conduced sensitivity analyses to explore the impact of methodological decisions within

\begin{tabular}{|c|c|c|c|c|c|}
\hline Author(s) and Year & ${ }_{\text {DKA }}{ }^{S}$ & ${ }^{12}$ Total & DKA $\stackrel{\text { Placebo }}{\text { Total }}$ & & Relative Risk [95\% Cl] \\
\hline Barnett, 2014 & 0 & 419 & 319 & 4 & 0.25 (0.01 to 6.21$)$ \\
\hline Bode, 2015 & 1 & 477 & 237 & $\rightarrow$ & 1.49 (0.06 to 36.53$)$ \\
\hline Dagogo-Jack, 2017 & 0 & 309 & 153 & $\longleftarrow$ & 0.50 (0.01 to 24.92$)$ \\
\hline Ikeda; 2015 & 0 & 261 & 67 & $\longleftarrow$ & $0.26(0.01$ to 12.96$)$ \\
\hline Inagaki, 2016 & 0 & 75 & 71 & $\longleftarrow$ & $0.95(0.02$ to 47.11$)$ \\
\hline Kadowaki, 2017 & 0 & 70 & 68 & 4 & 0.97 (0.02 to 48.29$)$ \\
\hline Nishimura, 2015 & 0 & 39 & 21 & 4 & 0.55 (0.01 to 26.77$)$ \\
\hline Rodbard, 2016 & 0 & 108 & 108 & 4 & 1.00 (0.02 to 49.95$)$ \\
\hline Roden, 2015 & 1 & 447 & 229 & 4 & 0.51 ( 0.03 to 8.15$)$ \\
\hline Rosenstock, 2014 & 1 & 375 & 188 & $\leftarrow$ & 0.50 (0.03 to 7.97$)$ \\
\hline Rosenstock, 2015 & 0 & 324 & 170 & $\leftarrow$ & 0.53 (0.01 to 26.40$)$ \\
\hline Rosenstock, 2017 & 0 & 412 & 209 & 4 & 0.51 (0.01 to 25.54$)$ \\
\hline Seino, 2014 & 0 & 223 & 57 & 4 & $0.26(0.01$ to 12.91$)$ \\
\hline Softeland, 2017 & 0 & 222 & 110 & $\leftarrow$ & 0.50 (0.01 to 24.92$)$ \\
\hline Tikkanen, 2015 & 0 & 552 & 272 & 4 & 0.16 (0.01 to 4.03$)$ \\
\hline Wilding; 2013 & 1 & 313 & 156 & $\rightarrow$ & $1.50(0.06$ to 36.61$)$ \\
\hline Yang, 2018 & 0 & 139 & 133 & $\rightarrow$ & $0.96(0.02$ to 47.89$)$ \\
\hline Zinman, 2015 & 4 & 4687 & 2333 & $\longrightarrow$ & $1.99(0.22$ to 17.80$)$ \\
\hline
\end{tabular}

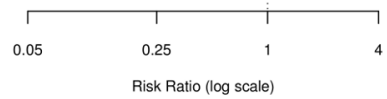

Figure 3 Risk of diabetic ketoacidosis (DKA) from sodium glucose co-transporter-2 (SGLT2) inhibitors compared with placebo. 


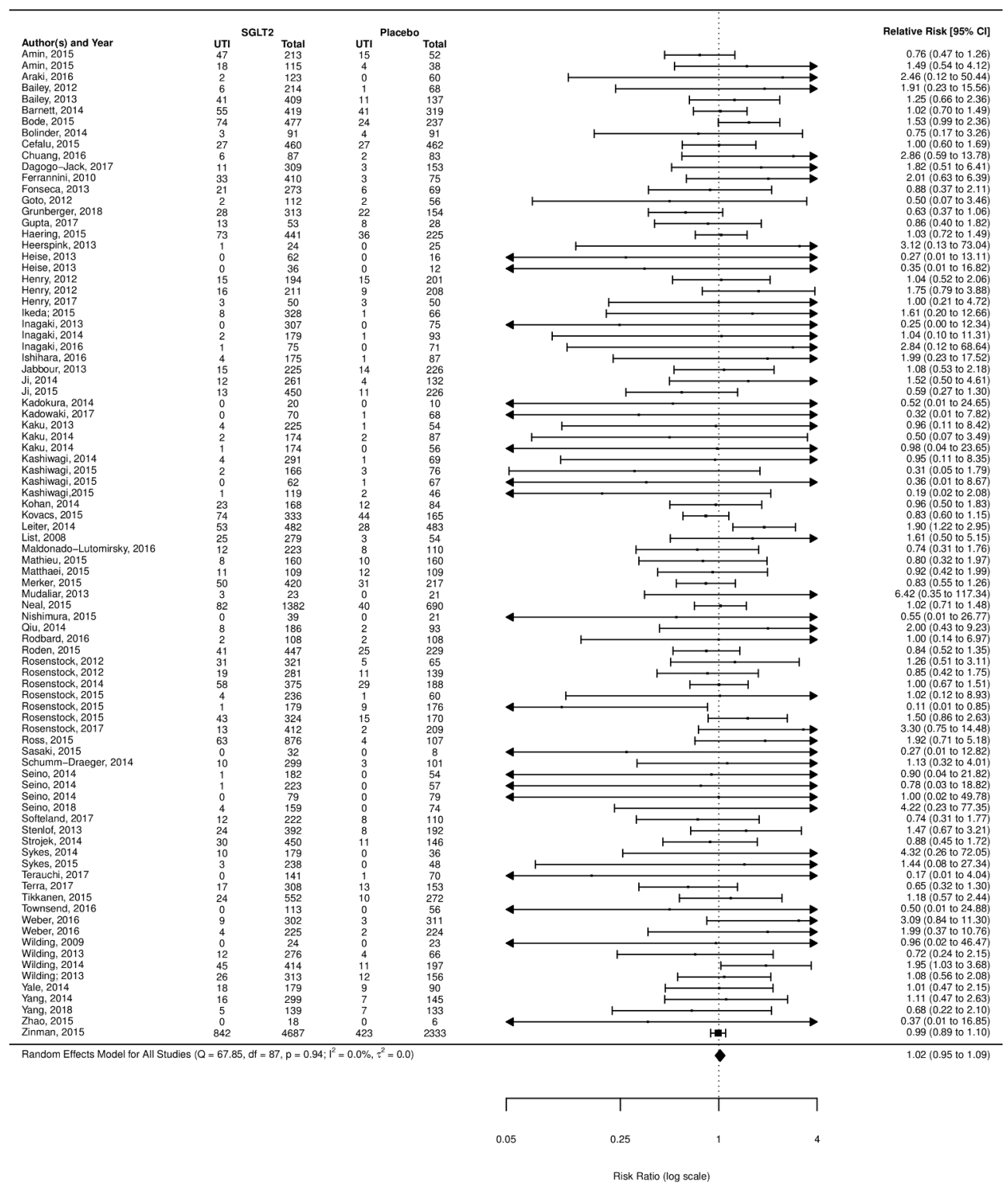

Figure 4 Risk of urinary tract infection (UTI) with sodium glucose co-transporter-2 (SGLT2) inhibitor compared with placebo.

our analysis. First, we pooled studies that had at least one reported event. Second, we repeated our analyses using fixed-effects models. All analyses were conducted using R statistical software (V.3.4.1). Technical online supplementary appendix, statistical code and dataset can be granted by contacting the corresponding author.

\section{RESULTS}

\section{Included studies}

A total of 2418 unique titles and abstracts were screened. Of these, 650 proceeded to full-text screening. A total of 143 citations met our inclusion criteria; however, 34 were excluded at the data extraction phase due to duplication of data, from the publication of extension studies or post hoc analyses. A final total of 109 publications were included, ${ }^{5} 23$ 35-141 representing 112 randomised populations (figure 1). Three publications reported on multiple unique populations. Most studies included one of the four marketed SGLT2 inhibitors, dapagliflozin (34 studies), canagliflozin (20 studies), empagliflozin (25 studies) and ipragliflozin (11 studies); while 21 studies included one of five non-marketed agents. With respect to comparators, 4 conducted within-class comparisons, 92 compared with placebo, 8 compared with metformin, 10 compared with an incretin agent, 5 compared with a sulfonylurea and 3 compared with pioglitizone. A total of nine studies included more than one unique comparator. One publication, reporting on the combined results of the CANVAS program ${ }^{23}$ studies only, provided events as rates per 1000 person-years. Data from this publication were only used for the amputation outcome assessment, data from an earlier publication on a subset of this population was used for other outcomes as actual event numbers were reported. ${ }^{82}$ Section 3 of the online supplementary appendix outlines the characteristics of each of the included studies. 


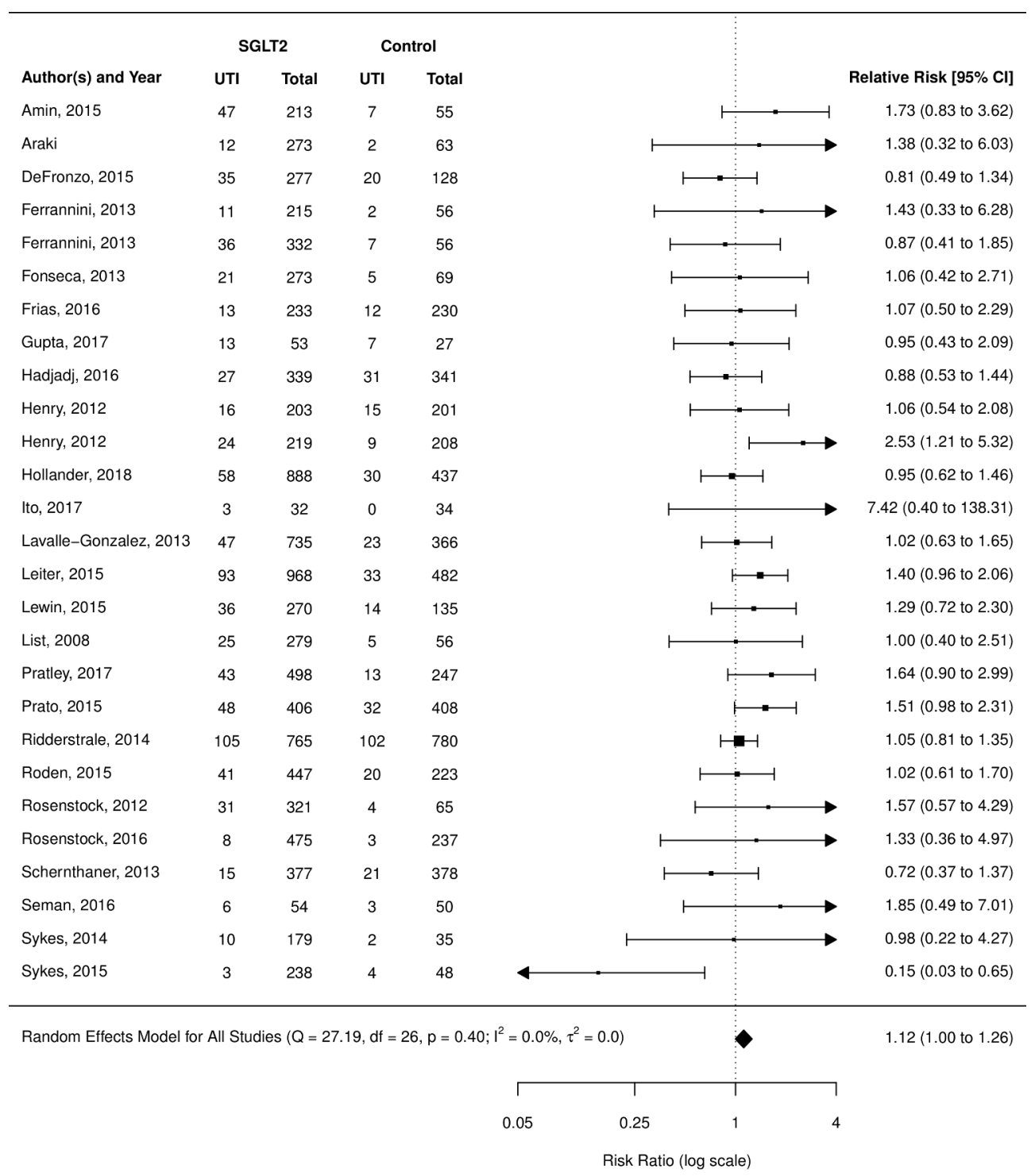

Figure 5 Risk of urinary tract infection (UTI) with sodium glucose co-transporter-2 (SGLT2) inhibitors compared with other active treatments.

\section{Primary analysis}

\section{Acute kidney injury}

AKI was reported in 11 RCTs (8 placebo comparison and 3 active comparison trials): meta-analysis was only possible with placebo-controlled trials. Overall, SGLT2 inhibitors were found to have a protective effect (RR $0.59 ; 95 \%$ CI 0.39 to $0.89, \mathrm{I}^{2}=0.0 \%$ ); however, this estimate is heavily weighted by one study using empagliflozin, the EMPA-REG trial (figure 2). ${ }^{5}$ Pooled estimate after removing the EMPA-REG trial was non-significant (RR $0.48 ; 95 \%$ CI 0.14 to $1.64 ; \mathrm{I}^{2}=0.0 \%$ ).

\section{Diabetic ketoacidosis}

DKA was reported in 26 RCTs (18 placebo comparison, 8 active comparisons and 1 within class comparison trial). Neither placebo (RR 0.66 ; $95 \%$ CI 0.30 to $1.45, \mathrm{I}^{2}=0.0 \%$ ) (figure 3) nor incretin (RR 0.43; 95\% CI 0.069 to 2.75; $\mathrm{I}^{2}=0.0 \%$; three studies) (forest plot, online supplementary appendix section 4) comparisons showed a significant difference in risk of DKA. Additional analysis using only placebo-controlled trials that had at least one event also yielded no significant difference (RR $0.73 ; 95 \%$ CI 0.25 to 2.16 ; $\mathrm{I}^{2}=0.0 \%$; seven studies) (forest plot, online supplementary appendix section 4 ).

\section{Urinary tract infections}

UTI was the most frequently reported outcome examined (110 of 112 studies reported). When compared with placebo, SGLT2 inhibitors as a class did not demonstrate a significant increased risk (RR 1.02; $95 \%$ CI 0.95 to 1.09 ) (figure 4); however, subgroup analysis of the individual agents did show a significantly increased risk of UTIs in users of dapagliflozin (RR 1.21; 95\% CI 1.02 to 1.43 ), but not empagliflozin, canagliflozin, ipragliflozin or non-marketed SGLT2 inhibitors (grouped) (supplementary appendix). When compared with active treatments, SGLT2 inhibitors grouped together did not demonstrate an 


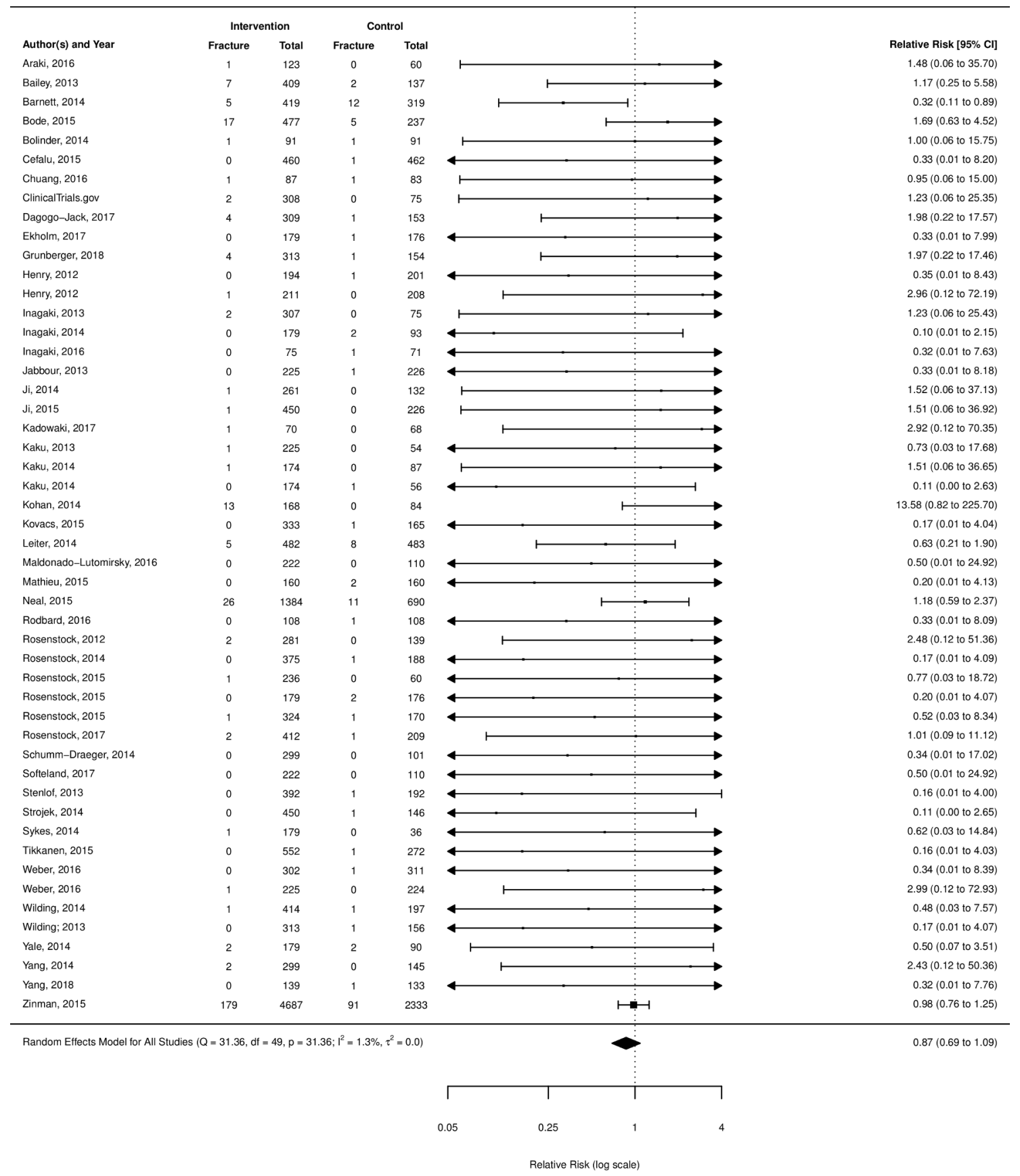

Figure 6 Risk of fracture with sodium glucose co-transporter-2 inhibitors compared with placebo.

increased risk of UTIs over metformin, sulfonylureas, incretins or glitizones (figure 5); however, when broken down by individual SGLT2 inhibitor, dapagliflozin showed an increased risk of UTI over active comparators grouped together (RR 1.42; 95\% CI 1.07 to 1.87 ) (forest plot, online supplementary appendix section 4).

Bone fracture

Bone fracture was reported in 63 RCTs (47 placebo comparisons, 14 active comparison and 2 within class comparisons). SGLT2 inhibitors were not found to have an increased risk of fractures over placebo (RR 0.87 ; 95\% CI 0.69 to 1.09 ) (figure 6), metformin
(RR $0.69 ; 95 \%$ CI 0.19 to $2.51 ; I^{2}=0.0 \%$; six studies), sulfonylureas (RR $1.15 ; 95 \%$ CI 0.66 to $2.00 ; \mathrm{I}^{2}=0.0 \%$; three studies) or incretins (RR 1.38; 95\% CI 0.31 to $6.17 ; \mathrm{I}^{2}=0.0 \%$; three studies). A subgroup analysis of canagliflozin compared with placebo alone, the agent identified by the FDA as having an increased risk, was also non-significant (RR 1.02; 95\% CI 0.63 to 1.65; $\mathrm{I}^{2}=0.0 \% ; 12$ studies) (additional forest plots, online supplementary appendix section 4).

Lower limb amputation

Data were identified on amputation for three studies. ${ }^{23} 46107$ One case of amputation was found in the ClinicalTrials.gov data for trial number NCT01422876 
in a user of empagliflozin $25 \mathrm{mg}$, no cases were reported for other treatment groups. The second study reported data from the CANVAS program, showed a rate of amputation among users of canagliflozin $(100-300 \mathrm{mg})$ was 6.3 per 1000 patient-years, compared with 3.4 per 1000 patient-years for placebo, this difference was statistically significant $(p<0.001)$. Actual number of events were not reported. The third study reported one case in each of the treatment groups, ertugliflozin $(1 / 888)$ and glimepiride $(1 / 437)$.
Subgroup and sensitivity analyses

Several subgroup analyses were conducted to examine the impact of prior and concurrent use of other antidiabetic agents; the influence of risk of bias as per the quality appraisal and the impact of the definition of UTI used as outlined in table 1 . Overall, these additional analyses did not change the findings of the primary analysis. There was a decreased risk of AKI in the treatment-naïve group, and the low risk of bias group, but this was consistent with the main analysis and driven by the same one

Table 1 Subgroup analysis among placebo-controlled trials

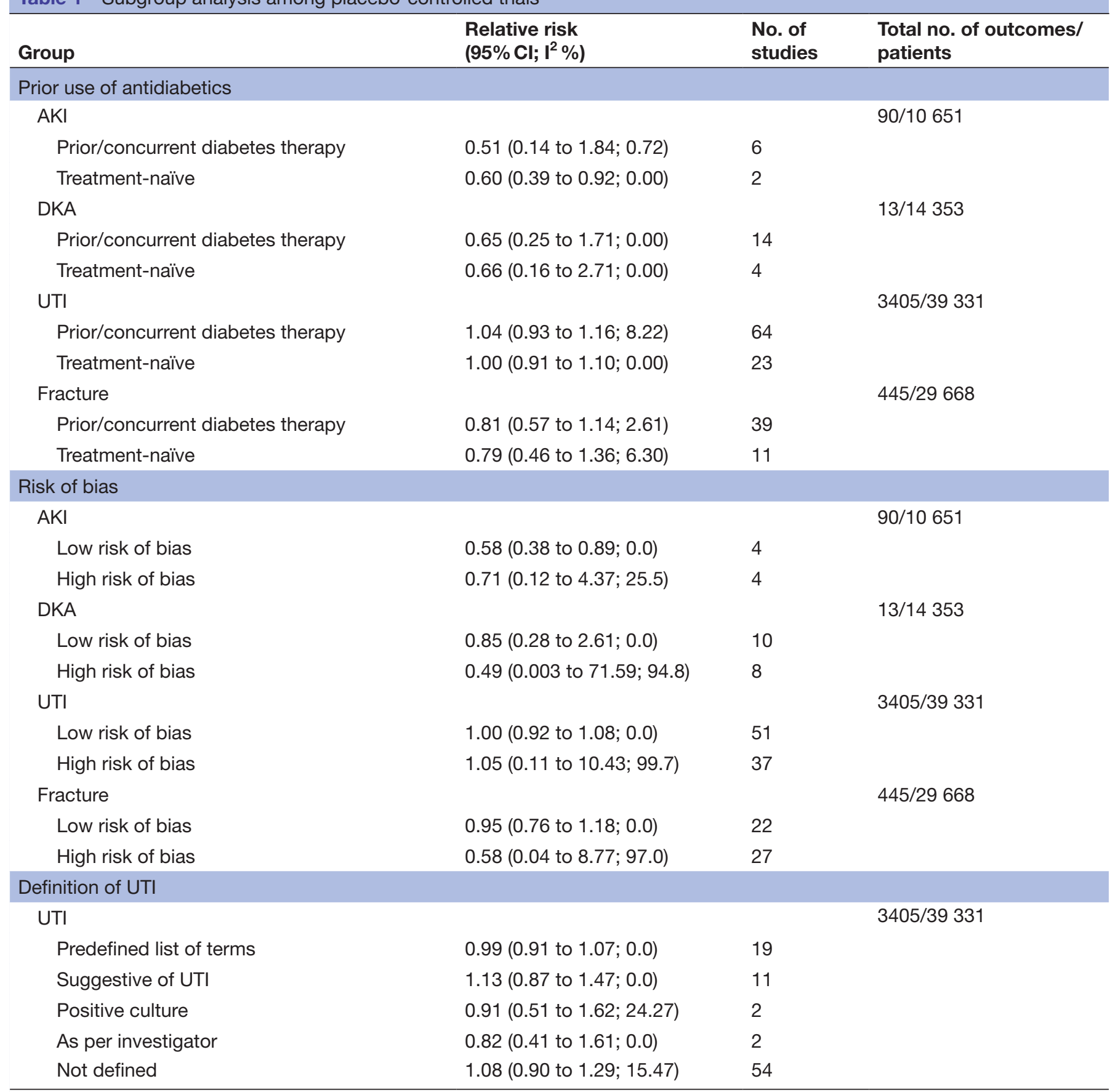

AKI, acute kidney injury; DKA, diabetic ketoacidosis; UTI, urinary tract infection. 
large study. ${ }^{142}$ When the analyses were re-run using a fixed-effects model, the risk estimates remained the same or had slightly smaller CIs. Forest plots for the fixed-effects analysis are mentioned in section 5 of the online supplementary appendix.

\section{Risk of bias}

Generally, studies were of good methodological quality; however, numerous studies were deemed high risk of selective reporting after outcome data were retrieved from ClinicalTrials.gov that were not reported in the peer-reviewed publication (28\%). Other potential sources of bias came from unclear reporting of methodological processes like randomisation sequence $(32 \%)$ or blinded outcome assessment $(17 \%)$, while most sources of bias came from lack of blinding of the researchers and participants (13\%) and of the outcome assessors (9\%). Risk of bias assessment for individual studies are available in section 6 of the online supplementary appendix. Funnel plots do not suggest the presence of publication bias (see section 7 of the online supplementary appendix).

\section{DISCUSSION}

This study provides a comprehensive review of the RCT literature with respect to key safety outcomes identified through postmarketing surveillance systems and communicated to health professionals and the public by drug regulators. We pooled outcome data from over 100 RCTs (including unpublished data only available through ClinicalTrials.gov) to quantify the association between SGLT2 inhibitors and AKI, DKA, UTI and bone fracture. We found that SGLT2 inhibitors as a class do not appear to increase the risk of DKA, UTI and bone fracture, and may have a protective effect with respect to AKI, although this effect was heavily weighted by one large RCT. With respect to UTI, overall findings do not hold in subgroup analysis by individual drug, suggesting that increased risk of UTI is associated only with dapagliflozin.

Despite early indication of a protective effect from SGLT2 inhibitors on kidney function, ${ }^{15}$ the FDA published in a safety communication in June 2016 that 101 cases of AKI were reported among users of canagliflozin and dapagliflozin. ${ }^{12}$ SGLT2 inhibitors may provide a longterm protective effect on the kidneys via reduced transglomerular pressure, similar to the effects of agents that target the renin-angiotensin-aldosterone (RAAS) axis. ${ }^{143}$ Szalat et al proposed three possible mechanisms that may explain the potential for an increased risk of AKI with SGLT2 inhibitors: (1) excessive diuresis leading to volume depletion, a particular concern for those who are haemodynamically unstable and volume-depleted; (2) a greater drop in transglomerular pressure due to the concomitant action of SGLT2 inhibition and RAAS blockade and (3) renal medullary hypoxic injury, likely occurring in patients taking concomitant agents that impair medullary oxygenation (eg, non-steroidal anti-inflammatory drugs, radio-contrast dyes). ${ }^{143}$ Additional potential mechanisms of renal injury include an increase in the urinary uric acid level leading to both crystal-dependent and crystal-independent tubular injury, and activation of aldose reductase resulting in fructose generation ultimately leading to increased oxidative stress, uric acid, cytokine release and inflammation. ${ }^{144}$ This systematic review highlights a lack of reporting of AKI with only 11 of 111 randomised comparisons having published data on this outcome. Although an overall protective effect was found, this finding was driven by one large RCT that compared empagliflozin with placebo. Evidence to support or refute the potential risk of AKI with use of canagliflozin or dapagliflozin was insufficient. Case reports filed with the FDA suggest that this adverse outcome frequently occurs early in therapy (within 1 month of initiation) and therefore this lack or reporting should not be due to the duration of clinical trials. Recent observational data also support clinical trial data on AKI. Nadkarni $e t$ al reported on the incidence of AKI among two cohorts comparing patients with type 2 diabetes using SGLT2 inhibitors to non-users. ${ }^{145}$ After an average follow-up time of 14 months, adjusted HR (aHR) showed SGLT2 inhibitors to be protective in one cohort (aHR 0.4 (95\% CI 0.2 to 0.7 ); $\mathrm{p}=0.004$ ) and favouring SGLT2 inhibitors, although not statistically significant, in the second cohort (aHR 0.6 (95\% CI 0.4 to 1.1$)$; $\mathrm{p}=0.09$ ). These findings were not driven by users of empagliflozin, rather $91.2 \%$ and $71.4 \%$ of SGLT2 inhibitor users in these cohorts were taking either canagliflozin or dapagliflozin, respectively.

Reports of euglycaemic DKA among patients with type 2 diabetes is concerning, as a diagnosis can easily be missed. Although rare, the SGLT2 inhibitors are thought to increase the risk by two potential mechanisms: (1) they increase urinary glucose excretion which leads to a reduction in insulin secretion and stimulates free fatty acid production which are later converted to ketone bodies and (2) they stimulate glucagon secretion which may lead to an overproduction of ketone bodies. ${ }^{146}$ An accurate assessment of the potential increased risk of DKA among users of SGLT2 inhibitors was difficult with the data reported within RCTs. Baseline incidence rates of DKA in patients with type 2 diabetes was found to be 1.34 per 1000 person-years in a 20-year retrospective Danish cohort study, with declining incidence each year. ${ }^{147}$ Therefore, most RCTs had insufficient sample size to detect any cases. Of the 16 RCTs that reported DKA, only 7 (representing 11004 patients) had one or more cases. Our findings are consistent with published observational literature, which indicates no increased risk, however CIs were wide. A case-control study using Truven MarketScan data (a large US claims database), ${ }^{148}$ and a cross-sectional using the FDA Adverse Event Reporting System database ${ }^{149}$ examining this issue have recently been published. Both studies used DPP-4 inhibitors as the active comparator given they have no known risk for DKA and are used in a similar fashion as second-line therapy in type 2 diabetes, and both showed significant increased risk with SGLT2 inhibitors (case-control: sevenfold increased risk among 140352 patients; cross-sectional: HR 2.2; 95\% CI 1.4 to 3.6, among 416 670). In contrast, the Danish cohort study did 
not find an increased risk of DKA in individuals taking SGLT2 inhibitors compared with other diabetes therapies (HR 1.6; 95\% CI 0.6 to 3.5), although the upper bound of the $95 \%$ CI does not rule out significant harm. ${ }^{147}$ No meta-analyses assessing this outcome were found.

Given the mechanism of action of the SGLT2 inhibitors, which work by inhibiting glucose reabsorption in the kidney leading to increase glucose excretion in the urine, an increased risk of UTI is plausible. In May 2015, the FDA reported in a safety update that 19 cases of life-threatening kidney or blood infections that originated as a UTI had been identified in patients taking a SGLT2 inhibitor. However, a meta-analysis published in 2017 included 77 RCTs representing 50820 patients and found no increased risk of UTIs in SGLT2 inhibitor users (RR 1.05; $95 \%$ CI 0.98 to 1.12$).{ }^{17}$ The previous meta-analysis limited inclusion to studies of at least 24 weeks and having a fulltext publication. Our study findings are consistent and add to the literature via the inclusion of 35 more studies, resulting in a more precise effect estimate. Importantly, subgroup analysis of individual SGLT2 inhibitors suggest variation of UTI risk within class, whereby dapagliflozin may increase UTI risk when compared with both placebo and active controls. A reasonable biologic mechanism for an increased risk of UTIs among dapagliflozin users is unclear; however, some early pathophysiological studies suggest that the dose-response relationship with urinary glucose excretion seems to plateau at the beginning of the normal recommended doses for most SGLT2 inhibitors, ${ }^{126} 136150-153$ although continues through the normal dosing range for dapagliflozin. ${ }^{154}$

In January 2016, the FDA issued an expanded warning regarding a potential increased risk for fracture with canagliflozin. ${ }^{9}$ A disruption in calcium-phosphate homeostasis is one potentially contributing mechanism. ${ }^{20}$ SGLT2 inhibitors increase serum phosphate levels via increased tubular reabsorption of phosphate. Increased phosphate levels then stimulate parathyroid hormone release which may enhance bone resorption leading to an increased fracture risk in patients using SGLT2 inhibitors. ${ }^{155}$ In an RCT conducted by Bode et al, additional investigation into the change in bone mineral density in canagliflozin versus placebo users was conducted. ${ }^{119}$ Their results showed a decreased placebo-corrected bone mineral density in the canagliflozin users at 2 years of $0.9 \%-1.2 \%$ at the hip, $0.3 \%-0.7 \%$ at the lumbar spine, $0.5 \%$ at the femoral neck and $0.4 \%$ at the distal forearm. Two meta-analyses have been published examining the risk of fracture when comparing SGLT2 inhibitors with placebo. ${ }^{2021}$ Ruanpeng et a $l^{20}$ included 20 RCTs, and Tang et al included 38 RCTs. Neither meta-analysis in pooled or subgroup analysis of individual SGLT2 inhibitors demonstrated a significant increased risk of fracture. A pooled analysis of eight canagliflozin RCTs also found no increased risk. ${ }^{22}$ The results of this current study support the existing literature, demonstrating risk neutrality, with the addition of new RCT literature (a total of 58 RCTs, 45 of which were placebo controlled).
To date, research evidence on the risk of amputations among users of SGLT2 inhibitors is limited to results from the combined CANVAS and CANVAS-R trials. Only two other studies reported amputations, with a combined total of three events. Further data are needed to establish the true risk as well as to identify if this may be a class effect or agent specific.

\section{Limitations}

Although we conducted a comprehensive systematic review of RCTs of SGLT2 inhibitors, there are still limitations to be considered when interpreting our findings. First, our review focused on select adverse events and excluded any benefits. Although this narrows the focus and requires the consideration of additional literature to make clinical decisions on appropriate use of SGLT2 inhibitors, it also provides a succinct and in-depth assessment of the unexpected adverse effects that have been reported postmarket. Second, several of the outcomes (eg, AKI, DKA, limb amputations) we evaluated occur infrequently. This also resulted in these individual outcomes to be at a higher risk of selective reporting bias than the more common adverse effects. We did our best to account for this risk by supplementing unreported outcomes with data from ClinicalTrials.gov; however, it is possible the cases of these outcomes were not recorded or reported through either of these sources. Third, certain outcomes may have been inadequately characterised within study reports. For example, while UTIs were commonly reported among RCTs included in this meta-analysis, data on complicated versus uncomplicated infections were not. The FDA highlighted 19 cases of life-threatening infections stemming from UTIs. It is possible that SGLT2 inhibitors play a role in the progression of UTI to more complicated clinical outcomes. Fourth, the limited duration of included RCTs ( $36 \%$ of studies were $<24$ weeks and $63 \%<1$ year) precludes the estimation of long-term effects of SGLT2 inhibitors. This may be important in case of declining bone integrity. Finally, it was difficult to accurately assess the methodological quality of the included studies given the fact we were examining secondary and rarely reported outcomes. It has been noted that traditional quality appraisal forms are not always well suited to systematic reviews of adverse events. This is due to the fact that sometimes data on adverse effects may be collected after allocation is known, or through self-assessment questionnaires. ${ }^{156}$

\section{CONCLUSION}

Despite the growing body of evidence on the new SGLT2 inhibitors, there remains minimal evidence demonstrating the comparative safety with respect to the more serious and unexpected outcomes. Current evidence from RCTs does not suggest an increased risk of harm with SGLT2 inhibitors, as a class, over placebo or active comparators with respect to the AKI, DKA, UTI or fracture. There appears to be treatment effect heterogeneity for the risk 
of UTI among specific SGLT2 inhibitors. Larger sample sizes and more long-term evidence, including observational studies, is needed to refine our estimates of the risk of AKI, DKA, fracture and amputation among SGLT2 inhibitor users.

Acknowledgements JRD holds a Fellowship from the Canadian Institutes of Health Research. JMG was supported by a New Investigator Award from the Canadian Institute of Health Research and a Clinician Scientist Award from the Canadian Diabetes Association.

Contributors JRD led the review and was involved at every stage, including protocol development, search strategy design, screening, data extraction, quality appraisal, analysis and manuscript preparation. CG was involved in screening, data extraction, quality appraisal and manuscript revisions and final approval. EC was involved in data cleaning and analysis, manuscript revisions and final approval. CAM was involved in project conception, protocol development and manuscript revisions and final approval. KAB was involved in project conception, protocol development and manuscript revisions and final approval. KJ was involved in project conception, protocol development and manuscript revisions and final approval. MS was involved in search strategy design, literature search and manuscript revisions and final approval. JH was involved in screening, data extraction, quality appraisal and manuscript revisions and final approval. DC was involved in screening, data extraction, quality appraisal and manuscript revisions and final approval. HN was involved in interpretation of study results, manuscript revisions and final approval. JMG supervised this research and was involved in protocol development, consensus on disagreements in data extraction, data analysis, interpretation of results, manuscript revisions and final approval.

Funding This work was partially supported by the Newfoundland and Labrador Centre for Applied Health Research.

Competing interests None declared.

Patient consent Not required.

Provenance and peer review Not commissioned; externally peer reviewed.

Data sharing statement All data used in this systematic review and metaanalysis are available through previously published articles and/or through clinical trials.gov. Section 2 of the supplementary appendix includes a complete list of data extraction variables that were collected. Access to the data can be granted by contacting the corresponding author.

Open access This is an open access article distributed in accordance with the Creative Commons Attribution Non Commercial (CC BY-NC 4.0) license, which permits others to distribute, remix, adapt, build upon this work non-commercially, and license their derivative works on different terms, provided the original work is properly cited, appropriate credit is given, any changes made indicated, and the use is non-commercial. See: http://creativecommons.org/licenses/by-nc/4.0/.

\section{REFERENCES}

1. Canadian Diabetes Association. 2016 Interim update to the guidelines: update to the pharmacologic management of Type 2 diabetes. 2016.

2. American Diabetes Association. Diabetes Guidelines Summary Recommendations from NDEI 12016 American Diabetes Association (ADA) Diabetes Guidelines Summary Recommendations from NDEl. 2016.

3. Marso SP, Daniels GH, Brown-Frandsen K, et al. Liraglutide and Cardiovascular Outcomes in Type 2 Diabetes. N Engl J Med Overseas Ed 2016;375:311-22.

4. Marso SP, Bain SC, Consoli A, et al. Semaglutide and Cardiovascular Outcomes in Patients with Type 2 Diabetes. N Engl J Med 2016;375:1834-44.

5. Zinman B, Wanner C, Lachin JM, et al. Empagliflozin, cardiovascular outcomes, and mortality in Type 2 diabetes. $N$ Engl $J$ Med Overseas Ed 2015;373:2117-28.

6. Mahaffey KW, Neal B, Perkovic V, et al. Canagliflozin for Primary and Secondary Prevention of Cardiovascular Events. Circulation 2018;137:323-34.

7. Health Canada. Summary Safety Review - Sodium Glucose Cotransporter 2 (SGLT2) Inhibitors INVOKANA (canagliflozin) and FORXIGA (dapagliflozin) - Evaluation of a Potential Risk of Acute
Kidney Injury. 2015 http://www.hc-sc.gc.ca/dhp-mps/medeff/ reviews-examens/sglt2-eng.php (Accessed 10 Dec 2015).

8. Health Canada Forxiga, Invokana. Health Canada begins safety review of diabetes drugs known as SGLT2 inhibitors and risk of ketoacidosis. http://healthycanadians.gc.ca/recall-alert-rappel-avis/ hc-sc/2015/53892a-eng.php (Accessed 10 Dec 2015).

9. Center for Drug Evaluation andResearch FDA Drug Safety Communication. FDA revises label of diabetes drug canagliflozin (Invokana, Invokamet) to include updates on bone fracture risk and new information on decreased bone mineral density. http://www. fda.gov/Drugs/DrugSafety/ucm461449.htm (Accessed 10 Dec 2015).

10. Center for Drug Evaluation and Research FDA Drug Safety Communication. FDA revises labels of SGLT2 inhibitors for diabetes to include warnings about too much acid in the blood and serious urinary tract infections. http://www.fda.gov/Drugs/DrugSafety/ ucm475463.htm (Accessed 10 Dec 2015).

11. Centre for Drug Evaluation and Research FDA Drug Safety Communication. FDA confirms increased risk of leg and foot amputations with the diabetes medicine canagliflozin (Invokana, Invokamet, Invokamet XR. https://www.fda.gov/Drugs/DrugSafety/ ucm557507.htm (Accessed 20 Dec 2017).

12. Centre for Drug Evaluation and Research FDA Drug Safety Communication. FDA strengthens kidney warnings for diabetes medicines canagliflozin (Invokana, Invokamet) and dapagliflozin (Farxiga, Xigduo XR). https://www.fda.gov/Drugs/DrugSafety/ ucm505860.htm (Accessed 12 Dec 2017).

13. European Medicines Agency. News and Events - SGLT2 inhibitors: information on potential risk of toe amputation to be included in prescribing information. http://www.ema.europa.eu/ema/index.jsp? curl=pages/news_and_events/news/2017/02/news_detail_002699. jsp\&mid=WC0b01ac058004d5c1 (Accessed 6 Feb 2018).

14. European Medicines Agency. EMA confirms recommendations to minimise ketoacidosis risk with SGLT2 inhibitors for diabetes. 2016 (Accessed 6 Feb 2018).

15. Fioretto $P$, Zambon $A$, Rossato $M$, et al. SGLT2 Inhibitors and the diabetic kidney. Diabetes Care 2016;39:S165-71.

16. Li D, Wang T, Shen S, et al. Urinary tract and genital infections in patients with type 2 diabetes treated with sodium-glucose cotransporter 2 inhibitors: a meta-analysis of randomized controlled trials. Diabetes Obes Metab 2017;19:348-55.

17. Liu J, Li L, Li S, et al. Effects of SGLT2 inhibitors on UTIs and genital infections in type 2 diabetes mellitus: a systematic review and meta-analysis. Sci Rep 2017;7:2824.

18. Zaccardi F, Webb DR, Htike ZZ, et al. Efficacy and safety of sodiumglucose co-transporter-2 inhibitors in type 2 diabetes mellitus: systematic review and network meta-analysis. Diabetes Obes Metab 2016;18:783-94.

19. Saad M, Mahmoud AN, Elgendy IY, et al. Cardiovascular outcomes with sodium-glucose cotransporter-2 inhibitors in patients with type II diabetes mellitus: a meta-analysis of placebo-controlled randomized trials. Int J Cardiol 2017;228:352-8.

20. Ruanpeng D, Ungprasert P, Sangtian J, et al. Sodium-glucose cotransporter 2 (SGLT2) inhibitors and fracture risk in patients with type 2 diabetes mellitus: A meta-analysis. Diabetes Metab Res Rev 2017;33:e2903.

21. Tang HL, Li DD, Zhang JJ, et al. Lack of evidence for a harmful effect of sodium-glucose co-transporter 2 (SGLT2) inhibitors on fracture risk among type 2 diabetes patients: a network and cumulative meta-analysis of randomized controlled trials. Diabetes Obes Metab 2016;18:1199-206.

22. Watts NB, Bilezikian JP, Usiskin K, et al. Effects of canagliflozin on fracture risk in patients with Type 2 diabetes mellitus. $J$ Clin Endocrinol Metab 2016;101:157-66.

23. Neal B, Perkovic V, Mahaffey KW, et al. Canagliflozin and Cardiovascular and Renal Events in Type 2 Diabetes. N Engl J Med Overseas Ed 2017;377:644-57.

24. Moher D, Liberati A, Tetzlaff J, et al. Preferred reporting items for systematic reviews and meta-analyses: the PRISMA statement. Ann Intern Med 2009;151:264-9.

25. Donnan J, Marra C, Aubrey Bassler K, et al. Comparative safety of the sodium glucose co-transporter 2 (SGLT2) inhibitors: a systematic review and network meta-analysis. 2017 PROSPERO 2016:CRD42016038715 Available from http://www.crd.york. ac.uk/PROSPERO_REBRANDING/display_record.asp?ID= CRD42016038715

26. Cochrane Handbook for Systematic Reviews of Interventions Version 5.1.0. 2011.

27. Hart B, Lundh A, Bero L. Effect of reporting bias on meta-analyses of drug trials: reanalysis of meta-analyses. BMJ 2012;344:d7202. 
28. Moher D, Pham B, Klassen TP, et al. What contributions do languages other than English make on the results of metaanalyses? J Clin Epidemiol 2000;53:964-72.

29. Morrison A, Polisena J, Husereau D, et al. The effect of Englishlanguage restriction on systematic review-based meta-analyses: a systematic review of empirical studies. Int J Technol Assess Health Care 2012;28:138-44.

30. Distiller SR. Evidence partners. Ottawa, Canada.

31. Khangura S, Konnyu K, Cushman R, et al. Evidence summaries: the evolution of a rapid review approach. Syst Rev 2012;1:10.

32. Higgins JP, Altman DG, Gøtzsche PC, et al. The Cochrane Collaboration's tool for assessing risk of bias in randomised trials. BMJ 2011;343:d5928.

33. Veroniki AA, Jackson D, Viechtbauer W, et al. Methods to estimate the between-study variance and its uncertainty in meta-analysis. Res Synth Methods 2016;7:55-79.

34. Higgins JP, Thompson SG, Deeks JJ, et al. Measuring inconsistency in meta-analyses. BMJ 2003;327:557-60.

35. Ikeda S, Takano Y, Cynshi O, et al. A novel and selective sodiumglucose cotransporter-2 inhibitor, tofogliflozin, improves glycaemic control and lowers body weight in patients with type 2 diabetes mellitus. Diabetes Obes Metab 2015;17:984-93.

36. Kashiwagi $\mathrm{A}$, Takahashi $\mathrm{H}$, Ishikawa $\mathrm{H}$, et al. A randomized, double-blind, placebo-controlled study on long-term efficacy and safety of ipragliflozin treatment in patients with type 2 diabetes mellitus and renal impairment: results of the long-term ASP1941 safety evaluation in patients with type 2 diabetes with renal impairment (LANTERN) study. Diabetes Obes Metab 2015;17:152-60.

37. Wilding JP, Norwood P, T'joen C, et al. A study of dapagliflozin in patients with type 2 diabetes receiving high doses of insulin plus insulin sensitizers: applicability of a novel insulin-independent treatment. Diabetes Care 2009:32:1656-62.

38. Fonseca VA, Ferrannini E, Wilding JP, et al. Active- and placebocontrolled dose-finding study to assess the efficacy, safety, and tolerability of multiple doses of ipragliflozin in patients with type 2 diabetes mellitus. J Diabetes Complications 2013;27:268-73.

39. An efficacy, safety, and tolerability study for TA-7284 in patients with Type 2 diabetes. https://clinicaltrials.gov/ct2/show/NCT01022112 (Accessed 15 Dec 2017).

40. Weber MA, Mansfield TA, Cain VA, et al. Blood pressure and glycaemic effects of dapagliflozin versus placebo in patients with type 2 diabetes on combination antihypertensive therapy: a randomised, double-blind, placebo-controlled, phase 3 study. Lancet Diabetes Endocrinol 2016;4:211-20.

41. Amin NB, Wang X, Mitchell JR, et al. Blood pressure-lowering effect of the sodium glucose co-transporter-2 inhibitor ertugliflozin, assessed via ambulatory blood pressure monitoring in patients with type 2 diabetes and hypertension. Diabetes Obes Metab 2015; 17:805-8

42. Schernthaner G, Gross JL, Rosenstock J, et al. Canagliflozin compared with sitagliptin for patients with type 2 diabetes who do not have adequate glycemic control with metformin plus sulfonylurea: a 52-week randomized trial. Diabetes Care 2013;36:2508-15

43. Ji L, Han P, Liu Y, et al. Canagliflozin in Asian patients with type 2 diabetes on metformin alone or metformin in combination with sulphonylurea. Diabetes, Obesity and Metabolism 2015;17:23-31.

44. Leiter LA, Yoon KH, Arias P, et al. Canagliflozin provides durable glycemic improvements and body weight reduction over 104 weeks versus glimepiride in patients with type 2 diabetes on metformin: a randomized, double-blind, phase 3 study. Diabetes Care 2015;38:355-64.

45. Mudaliar S, Henry RR, Boden G, et al. Changes in insulin sensitivity and insulin secretion with the sodium glucose cotransporter 2 inhibitor dapagliflozin. Diabetes Technol Ther 2014;16:137-44.

46. DeFronzo RA, Lewin A, Patel S, et al. Combination of empagliflozin and linagliptin as second-line therapy in subjects with type 2 diabetes inadequately controlled on metformin. Diabetes Care 2015;38:384-93.

47. Ekholm E, Hansen L, Johnsson E, et al. Combined treatment with saxagliptin plus dapagliflozin reduces insulin levels by increased insulin clearance and improves $\beta$-Cell function. Endocr Pract 2017;23:258-65.

48. Ridderstråle M, Andersen KR, Zeller $\mathrm{C}$, et al. Comparison of empagliflozin and glimepiride as add-on to metformin in patients with type 2 diabetes: a 104-week randomised, activecontrolled, double-blind, phase 3 trial. Lancet Diabetes Endocrinol 2014;2:691-700.

49. Ito D, Shimizu S, Inoue K, et al. Comparison of ipragliflozin and pioglitazone effects on nonalcoholic fatty liver disease in patients with Type 2 diabetes: a randomized, 24-week, open-label, activecontrolled trial. Diabetes Care 2017; 40:1364-72.

50. Yang L, Di G, Qi X, et al. Substance P promotes diabetic corneal epithelial wound healing through molecular mechanisms mediated via the neurokinin-1 receptor. Diabetes 2014;63:4262-74.

51. Lambers Heerspink HJ, de Zeeuw D, Wie L, et al. Dapagliflozin a glucose-regulating drug with diuretic properties in subjects with type 2 diabetes. Diabetes Obes Metab 2013;15:853-62.

52. Bailey CJ, Gross JL, Hennicken D, et al. Dapagliflozin add-on to metformin in type 2 diabetes inadequately controlled with metformin: a randomized, double-blind, placebo-controlled 102week trial. BMC Med 2013;11:43.

53. Strojek K, Yoon KH, Hruba V, et al. Dapagliflozin added to glimepiride in patients with type 2 diabetes mellitus sustains glycemic control and weight loss over 48 weeks: a randomized, double-blind, parallel-group, placebo-controlled trial. Diabetes Ther 2014;5:267-83.

54. Leiter LA, Cefalu WT, de Bruin TW, et al. Dapagliflozin added to usual care in individuals with type 2 diabetes mellitus with preexisting cardiovascular disease: a 24-week, multicenter, randomized, double-blind, placebo-controlled study with a 28-week extension. J Am Geriatr Soc 2014;62:1252-62.

55. Yang W, Ma J, Li Y, et al. Dapagliflozin as add-on therapy in Asian patients with type 2 diabetes inadequately controlled on insulin with or without oral antihyperglycemic drugs: a randomized controlled trial. J Diabetes 2018;10:589-99.

56. Ji L, Ma J, Li H, et al. Dapagliflozin as monotherapy in drug-naive Asian patients with type 2 diabetes mellitus: a randomized, blinded, prospective phase III study. Clin Ther 2014;36:84-100.

57. Wilding JP, Woo V, Rohwedder K, et al. Dapagliflozin in patients with type 2 diabetes receiving high doses of insulin: efficacy and safety over 2 years. Diabetes Obes Metab 2014;16:124-36.

58. Bolinder J, Ljunggren Ö, Johansson L, et al. Dapagliflozin maintains glycaemic control while reducing weight and body fat mass over 2 years in patients with type 2 diabetes mellitus inadequately controlled on metformin. Diabetes Obes Metab 2014;16:159-69.

59. Bailey CJ, lqbal N, T'joen C, et al. Dapagliflozin monotherapy in drug-naïve patients with diabetes: a randomized-controlled trial of low-dose range. Diabetes Obes Metab 2012;14:951-9.

60. Ferrannini E, Ramos SJ, Salsali A, et al. Dapagliflozin monotherapy in type 2 diabetic patients with inadequate glycemic control by diet and exercise: a randomized, double-blind, placebo-controlled, phase 3 trial. Diabetes Care 2010;33:2217-24.

61. Henry RR, Murray AV, Marmolejo MH, et al. Dapagliflozin, metformin $\mathrm{XR}$, or both: initial pharmacotherapy for type 2 diabetes, a randomised controlled trial. Int J Clin Pract 2012;66:446-56.

62. Cefalu WT, Leiter LA, de Bruin TW, et al. Dapagliflozin's effects on glycemia and cardiovascular risk factors in high-risk patients with Type 2 diabetes: a 24-week, multicenter, randomized, double-blind, placebo-controlled study with a 28 -week extension. Diabetes Care 2015;38:1218-27.

63. Seino Y, Sasaki T, Fukatsu A, et al. Dose-finding study of luseogliflozin in Japanese patients with type 2 diabetes mellitus: a 12-week, randomized, double-blind, placebo-controlled, phase II study. Curr Med Res Opin 2014;30:1231-44.

64. Rosenstock J, Aggarwal N, Polidori D, et al. Dose-ranging effects of canagliflozin, a sodium-glucose cotransporter 2 inhibitor, as addon to metformin in subjects with type 2 diabetes. Diabetes Care 2012;35:1232-8.

65. Amin NB, Wang X, Jain SM, et al. Dose-ranging efficacy and safety study of ertugliflozin, a sodium-glucose co-transporter 2 inhibitor, in patients with type 2 diabetes on a background of metformin. Diabetes Obes Metab 2015;17:591-8.

66. Rosenstock J, Hansen L, Zee P, et al. Dual add-on therapy in type 2 diabetes poorly controlled with metformin monotherapy: a randomized double-blind trial of saxagliptin plus dapagliflozin addition versus single addition of saxagliptin or dapagliflozin to metformin. Diabetes Care 2015;38:376-83.

67. Matthaei S, Bowering K, Rohwedder K, et al. Durability and tolerability of dapagliflozin over 52 weeks as add-on to metformin and sulphonylurea in type 2 diabetes. Diabetes Obes Metab 2015;17:1075-84

68. Nishimura R, Tanaka Y, Koiwai K, et al. Effect of empagliflozin monotherapy on postprandial glucose and 24-hour glucose variability in Japanese patients with type 2 diabetes mellitus: a randomized, double-blind, placebo-controlled, 4-week study. Cardiovasc Diabetol 2015;14:11

69. Rosenstock J, Frias J, Páll D, et al. Effect of ertugliflozin on glucose control, body weight, blood pressure and bone density in type 2 diabetes mellitus inadequately controlled on metformin monotherapy (VERTIS MET). Diabetes Obes Metab 2018;20:520-9. 
70. Henry R, Strange P, Zhou P, et al. Effects of dapagliflozin (DAPA), a sodium glucose cotransporter 2 inhibitor, on 24-hour glycemic control in patients with type 2 diabetes (T2D) Henry RR, Strange P, Zhou R, Zhuplatov SB, Mansfield T, Klein D and Katz A. 2016;65:A311.

71. Weber MA, Mansfield TA, Alessi F, et al. Effects of dapagliflozin on blood pressure in hypertensive diabetic patients on reninangiotensin system blockade. Blood Press 2016;25:93-103.

72. Rosenstock J, Vico M, Wei L, et al. Effects of dapagliflozin, an SGLT2 inhibitor, on $\mathrm{HbA}(1 \mathrm{c})$, body weight, and hypoglycemia risk in patients with type 2 diabetes inadequately controlled on pioglitazone monotherapy. Diabetes Care 2012;35:1473-8.

73. Inagaki $\mathrm{N}$, Kondo K, Yoshinari T, Kuki H. Efficacy and safety of canagliflozin alone or as add-on to other oral antihyperglycemic drugs in Japanese patients with type 2 diabetes: A 52-week openlabel study. J Diabetes Investig 2015;6:210-8.

74. Kadowaki T, Inagaki N, Kondo K, et al. Efficacy and safety of canagliflozin as add-on therapy to teneligliptin in Japanese patients with type 2 diabetes mellitus: Results of a 24-week, randomized, double-blind, placebo-controlled trial. Diabetes Obes Metab 2017;19:874-82.

75. Lavalle-González FJ, Januszewicz A, Davidson J, et al. Efficacy and safety of canagliflozin compared with placebo and sitagliptin in patients with type 2 diabetes on background metformin monotherapy: a randomised trial. Diabetologia 2013;56:2582-92.

76. Inagaki N, Harashima S, Maruyama N, et al. Efficacy and safety of canagliflozin in combination with insulin: a double-blind, randomized, placebo-controlled study in Japanese patients with type 2 diabetes mellitus. Cardiovasc Diabetol 2016;15:89.

77. Inagaki N, Kondo K, Yoshinari T, et al. Efficacy and safety of canagliflozin in Japanese patients with type 2 diabetes: a randomized, double-blind, placebo-controlled, 12-week study. Diabetes Obes Metab 2013;15:1136-45.

78. Wilding JP, Charpentier G, Hollander P, et al. Efficacy and safety of canagliflozin in patients with type 2 diabetes mellitus inadequately controlled with metformin and sulphonylurea: a randomised trial. Int J Clin Pract 2013;67:1267-82.

79. Inagaki N, Kondo K, Yoshinari T, et al. Efficacy and safety of canagliflozin monotherapy in Japanese patients with type 2 diabetes inadequately controlled with diet and exercise: a 24-week, randomized, double-blind, placebo-controlled, Phase III study. Expert Opin Pharmacother 2014;15:1501-15.

80. Stenlöf K, Cefalu WT, Kim KA, et al. Efficacy and safety of canagliflozin monotherapy in subjects with type 2 diabetes mellitus inadequately controlled with diet and exercise. Diabetes Obes Metab 2013:15:372-82.

81. Yale JF, Bakris G, Cariou B, et al. Efficacy and safety of canagliflozin over 52 weeks in patients with type 2 diabetes mellitus and chronic kidney disease. Diabetes Obes Metab 2014; $16: 1016-27$

82. Neal B, Perkovic V, de Zeeuw D, et al. Efficacy and safety of canagliflozin, an inhibitor of sodium-glucose cotransporter 2, when used in conjunction with insulin therapy in patients with type 2 diabetes. Diabetes Care 2015;38:403-11.

83. Kaku K, Inoue S, Matsuoka O, et al. Efficacy and safety of dapagliflozin as a monotherapy for type 2 diabetes mellitus in Japanese patients with inadequate glycaemic control: a phase II multicentre, randomized, double-blind, placebo-controlled trial. Diabetes Obes Metab 2013;15:432-40.

84. Araki E, Onishi Y, Asano M, et al. Efficacy and safety of dapagliflozin in addition to insulin therapy in Japanese patients with type 2 diabetes: Results of the interim analysis of 16-week double-blind treatment period. J Diabetes Investig 2016;7:555-64.

85. Kaku K, Kiyosue A, Inoue S, et al. Efficacy and safety of dapagliflozin monotherapy in Japanese patients with type 2 diabetes inadequately controlled by diet and exercise. Diabetes Obes Metab 2014:16:1102-10.

86. Barnett AH, Mithal A, Manassie J, et al. Efficacy and safety of empagliflozin added to existing antidiabetes treatment in patients with type 2 diabetes and chronic kidney disease: a randomised, double-blind, placebo-controlled trial. Lancet Diabetes Endocrinol 2014;2:369-84.

87. Kadowaki T, Haneda M, Inagaki N, et al. Efficacy and safety of empagliflozin monotherapy for 52 weeks in Japanese patients with type 2 diabetes: a randomized, double-blind, parallel-group study. Adv Ther 2015;32:306-18

88. Ross S, Thamer C, Cescutti J, et al. Efficacy and safety of empagliflozin twice daily versus once daily in patients with type 2 diabetes inadequately controlled on metformin: a 16-week, randomized, placebo-controlled trial. Diabetes Obes Metab 2015;17:699-702
89. Ishihara $\mathrm{H}$, Yamaguchi $\mathrm{S}$, Nakao I, et al. Efficacy and safety of ipragliflozin as add-on therapy to insulin in Japanese patients with type 2 diabetes mellitus (IOLITE): a multi-centre, randomized, placebo-controlled, double-blind study. Diabetes Obes Metab 2016;18:1207-16.

90. Kashiwagi A, Akiyama N, Shiga T, et al. Efficacy and safety of ipragliflozin as an add-on to a sulfonylurea in Japanese patients with inadequately controlled type 2 diabetes: results of the randomized, placebo-controlled, double-blind, phase III EMIT study. Diabetol Int 2015;6:125-38.

91. Wilding JP, Ferrannini E, Fonseca VA, et al. Efficacy and safety of ipragliflozin in patients with type 2 diabetes inadequately controlled on metformin: a dose-finding study. Diabetes Obes Metab 2013;15:403-9.

92. Seino Y, Sasaki T, Fukatsu A, et al. Efficacy and safety of luseogliflozin added to insulin therapy in Japanese patients with type 2 diabetes: a multicenter, 52-week, clinical study with a 16week, double-blind period and a 36-week, open-label period. Curr Med Res Opin 2018;34:981-94.

93. Seino $Y$, Sasaki T, Fukatsu $A$, et al. Efficacy and safety of luseogliflozin as monotherapy in Japanese patients with type 2 diabetes mellitus: a randomized, double-blind, placebo-controlled, phase 3 study. Curr Med Res Opin 2014;30:1245-55.

94. Seino Y, Sasaki T, Fukatsu A, et al. Efficacy and safety of luseogliflozin monotherapy in Japanese patients with type 2 diabetes mellitus: a 12-week, randomized, placebo-controlled, phase II study. Curr Med Res Opin 2014;30:1219-30.

95. Kaku K, Watada H, Iwamoto Y, et al. Efficacy and safety of monotherapy with the novel sodium/glucose cotransporter-2 inhibitor tofogliflozin in Japanese patients with type 2 diabetes mellitus: a combined Phase 2 and 3 randomized, placebocontrolled, double-blind, parallel-group comparative study. Cardiovasc Diabetol 2014:13:65

96. Dagogo-Jack S, Liu J, Eldor R, et al. Efficacy and safety of the addition of ertugliflozin in patients with type 2 diabetes mellitus inadequately controlled with metformin and sitagliptin: The VERTIS SITA2 placebo-controlled randomized study. Diabetes Obes Metab 2018;20.

97. Rodbard HW, Seufert J, Aggarwal N, et al. Efficacy and safety of titrated canagliflozin in patients with type 2 diabetes mellitus inadequately controlled on metformin and sitagliptin. Diabetes Obes Metab 2016;18:812-9.

98. Terauchi $\mathrm{Y}$, Tamura M, Senda M, et al. Efficacy and safety of tofogliflozin in Japanese patients with type 2 diabetes mellitus with inadequate glycaemic control on insulin therapy (J-STEP/INS): Results of a 16-week randomized, double-blind, placebo-controlled multicentre trial. Diabetes Obes Metab 2017;19:1397-407.

99. Qiu R, Capuano G, Meininger G. Efficacy and safety of twice-daily treatment with canagliflozin, a sodium glucose co-transporter 2 inhibitor, added on to metformin monotherapy in patients with type 2 diabetes mellitus. J Clin Trans/ Endocrinol 2014;1:54-60.

100. Lu CH, Min KW, Chuang LM, et al. Efficacy, safety, and tolerability of ipragliflozin in Asian patients with type 2 diabetes mellitus and inadequate glycemic control with metformin: Results of a phase 3 randomized, placebo-controlled, double-blind, multicenter trial. $J$ Diabetes Investig 2016;7:366-73.

101. Maldonado-Lutomirsky M, SÃ fteland E, Meier J, et al. Empagliflozin (EMPA) as add-on to linagliptin (LINA) and metformin in patients with type 2 diabetes (T2DM): A 24-week randomised, double-blind, parallel-group trial. Diabetologia 2016;59:S93

102. Søfteland E, Meier JJ, Vangen B, et al. Empagliflozin as add-on therapy in patients with Type 2 diabetes inadequately controlled with linagliptin and metformin: a 24-week randomized, doubleblind, parallel-group trial. Diabetes Care 2017;40:201-9.

103. Kovacs CS, Seshiah V, Merker L, et al. Empagliflozin as add-on therapy to pioglitazone with or without metformin in patients with Type 2 diabetes mellitus. Clin Ther 2015;37:1773-88.

104. Merker L, Häring HU, Christiansen AV, et al. Empagliflozin as add-on to metformin in people with Type 2 diabetes. Diabet Med 2015;32:1555-67.

105. Haering HU, Merker L, Christiansen AV, et al. Empagliflozin as addon to metformin plus sulphonylurea in patients with type 2 diabetes. Diabetes Res Clin Pract 2015;110:82-90.

106. Tikkanen I, Narko K, Zeller C, et al. Empagliflozin reduces blood pressure in patients with type 2 diabetes and hypertension. Diabetes Care 2015;38:420-8.

107. Hollander P, Liu J, Hill J, et al. Ertugliflozin compared with glimepiride in patients with Type 2 diabetes mellitus inadequately controlled on metformin: the VERTIS SU randomized study. Diabetes Ther 2018:9:193-207. 
108. Grunberger G, Camp S, Johnson J, et al. Ertugliflozin in patients with stage 3 chronic kidney disease and Type 2 diabetes mellitus: the vertis renal randomized study. Diabetes Ther 2018;9:49-66.

109. Pratley RE, Eldor R, Raji A, et al. Ertugliflozin plus sitagliptin versus either individual agent over 52 weeks in patients with type 2 diabetes mellitus inadequately controlled with metformin: The VERTIS FACTORIAL randomized trial. Diabetes Obes Metab 2018;20:1111-20

110. Frías JP, Guja C, Hardy E, et al. Exenatide once weekly plus dapagliflozin once daily versus exenatide or dapagliflozin alone in patients with type 2 diabetes inadequately controlled with metformin monotherapy (DURATION-8): a 28 week, multicentre, double-blind, phase 3, randomised controlled trial. Lancet Diabetes Endocrinol 2016;4:1004-16.

111. Rosenstock J, Cefalu WT, Lapuerta P, et al. Greater dose-ranging effects on A1C levels than on glucosuria with LX4211, a dual inhibitor of SGLT1 and SGLT2, in patients with type 2 diabetes on metformin monotherapy. Diabetes Care 2015;38:431-8.

112. Rosenstock J, Jelaska A, Zeller C, et al. Impact of empagliflozin added on to basal insulin in type 2 diabetes inadequately controlled on basal insulin: a 78-week randomized, double-blind, placebocontrolled trial. Diabetes Obes Metab 2015;17:936-48.

113. Rosenstock J, Jelaska A, Frappin G, et al. Improved glucose control with weight loss, lower insulin doses, and no increased hypoglycemia with empagliflozin added to titrated multiple daily injections of insulin in obese inadequately controlled type 2 diabetes. Diabetes Care 2014;37:1815-23.

114. Lewin A, DeFronzo RA, Patel S, et al. Initial combination of empagliflozin and linagliptin in subjects with type 2 diabetes. Diabetes Care 2015;38:394-402.

115. Hadjadj S, Rosenstock J, Meinicke T, et al. Initial combination of empagliflozin and metformin in patients with Type 2 diabetes. Diabetes Care 2016;39:1718-28.

116. Rosenstock J, Chuck L, González-Ortiz M, et al. Initial combination therapy with canagliflozin plus metformin versus each component as monotherapy for drug-naïve Type 2 diabetes. Diabetes Care 2016;39:353-62.

117. Kashiwagi A, Kazuta K, Takinami Y, et al. Ipragliflozin improves glycemic control in Japanese patients with type 2 diabetes mellitus: the BRIGHTEN study: BRIGHTEN: double-blind randomized study of ipragliflozin to show its efficacy as monotherapy in T2DM patients. Diabetol Int 2015;6:8-18.

118. Goto K, Kashiwagi A, Kazuta K. Ipragliflozin reduces A1C and body weight in type 2 diabetes patients who have inadequate glycemic control on metformin alone: Illuminate study. Diabetes 2012;61:A212-344.

119. Bode B, Stenlöf K, Harris S, et al. Long-term efficacy and safety of canagliflozin over 104 weeks in patients aged 55-80 years with type 2 diabetes. Diabetes Obes Metab 2015;17:294-303.

120. Gupta S, Shaikh S, Joshi P, et al. Long-Term Efficacy and Safety of Empagliflozin Monotherapy in Drug-Naïve Patients with Type 2 Diabetes in Indian Subgroup: Results from a 76-week Extension Trial of Phase III, Double-Blind, Randomized Study. Indian J Endocrinol Metab 2017;21:286.

121. Del Prato S, Nauck M, Durán-Garcia S, et al. Long-term glycaemic response and tolerability of dapagliflozin versus a sulphonylurea as add-on therapy to metformin in patients with type 2 diabetes: 4-year data. Diabetes Obes Metab 2015;17:581-90.

122. Ferrannini E, Berk A, Hantel S, et al. Long-term safety and efficacy of empagliflozin, sitagliptin, and metformin: an active-controlled, parallel-group, randomized, 78-week open-label extension study in patients with type 2 diabetes. Diabetes Care 2013;36:4015-21.

123. Tanizawa Y, Kaku K, Araki E, et al. Long-term safety and efficacy of tofogliflozin, a selective inhibitor of sodium-glucose cotransporter 2 , as monotherapy or in combination with other oral antidiabetic agents in Japanese patients with type 2 diabetes mellitus: multicenter, open-label, randomized controlled trials. Expert Opin Pharmacother 2014;15:749-66.

124. Kohan DE, Fioretto $P$, Tang W, et al. Long-term study of patients with type 2 diabetes and moderate renal impairment shows that dapagliflozin reduces weight and blood pressure but does not improve glycemic control. Kidney Int 2014;85:962-71.

125. Araki E, Tanizawa $Y$, Tanaka $Y$, et al. Long-term treatment with empagliflozin as add-on to oral antidiabetes therapy in Japanese patients with type 2 diabetes mellitus. Diabetes Obes Metab 2015;17:665-74.

126. Zhao X, Cui Y, Zhao S, et al. Pharmacokinetic and pharmacodynamic properties and tolerability of single- and multiple-dose once-daily empagliflozin, a sodium glucose cotransporter 2 inhibitor, in chinese patients with Type 2 diabetes mellitus. Clin Ther 2015;37:1493-502.
127. Kadokura T, Akiyama N, Kashiwagi A, et al. Pharmacokinetic and pharmacodynamic study of ipragliflozin in Japanese patients with type 2 diabetes mellitus: a randomized, double-blind, placebocontrolled study. Diabetes Res Clin Pract 2014;106:50-6.

128. Sasaki T, Seino Y, Fukatsu A, et al. Pharmacokinetics, Pharmacodynamics, and Safety of Luseogliflozin in Japanese Patients with Type 2 Diabetes Mellitus: A Randomized, Single-blind, Placebo-controlled Trial. Adv Ther 2015;32:319-40.

129. Terra SG, Focht K, Davies M, et al. Phase III, efficacy and safety study of ertugliflozin monotherapy in people with type 2 diabetes mellitus inadequately controlled with diet and exercise alone. Diabetes Obes Metab 2017:19:721-8.

130. Sykes AP, Kemp GL, Dobbins R, et al. Randomized efficacy and safety trial of once-daily remogliflozin etabonate for the treatment of type 2 diabetes. Diabetes Obes Metab 2015;17:98-101.

131. Sykes AP, O'Connor-Semmes R, Dobbins R, et al. Randomized trial showing efficacy and safety of twice-daily remogliflozin etabonate for the treatment of type 2 diabetes. Diabetes Obes Metab 2015;17:94-7.

132. Mathieu C, Ranetti AE, Li D, et al. Randomized, double-blind, phase 3 trial of triple therapy with dapagliflozin add-on to saxagliptin plus metformin in Type 2 diabetes. Diabetes Care 2015;38:2009-17.

133. Kashiwagi A, Kazuta K, Yoshida S, et al. Randomized, placebocontrolled, double-blind glycemic control trial of novel sodiumdependent glucose cotransporter 2 inhibitor ipragliflozin in Japanese patients with type 2 diabetes mellitus. J Diabetes Investig 2014:5:382-91.

134. Townsend RR, Machin I, Ren J, et al. Reductions in mean 24-Hour ambulatory blood pressure after 6-week treatment with canagliflozin in patients with Type 2 diabetes mellitus and hypertension. $J$ Clin Hypertens 2016;18:43-52.

135. Roden M, Merker L, Christiansen AV, et al. Safety, tolerability and effects on cardiometabolic risk factors of empagliflozin monotherapy in drug-naïve patients with type 2 diabetes: a double-blind extension of a Phase III randomized controlled trial. Cardiovasc Diabetol 2015;14:154.

136. Heise T, Seewaldt-Becker E, Macha S, et al. Safety, tolerability, pharmacokinetics and pharmacodynamics following 4 weeks' treatment with empagliflozin once daily in patients with type 2 diabetes. Diabetes Obes Metab 2013:15:613-21.

137. Heise T, Seman L, Macha S, et al. Safety, tolerability, pharmacokinetics, and pharmacodynamics of multiple rising doses of empagliflozin in patients with type 2 diabetes mellitus. Diabetes Ther 2013;4:331-45.

138. List JF, Woo V, Morales E, et al. Sodium-glucose cotransport inhibition with dapagliflozin in type 2 diabetes. Diabetes Care 2009;32:650-7

139. Wan Seman WJ, Kori N, Rajoo S, et al. Switching from sulphonylurea to a sodium-glucose cotransporter2 inhibitor in the fasting month of Ramadan is associated with a reduction in hypoglycaemia. Diabetes Obes Metab 2016;18:628-32.

140. Jabbour SA, Hardy E, Sugg J, et al. Dapagliflozin is effective as add-on therapy to sitagliptin with or without metformin: a 24-week, multicenter, randomized, double-blind, placebo-controlled study. Diabetes Care 2014;37:740-50.

141. Schumm-Draeger PM, Burgess L, Korányi L, et al. Twice-daily dapagliflozin co-administered with metformin in type 2 diabetes: a 16-week randomized, placebo-controlled clinical trial. Diabetes Obes Metab 2015;17:42-51.

142. Zinman B, Wanner C, Lachin JM, et al. Empagliflozin, cardiovascular outcomes, and mortality in Type 2 diabetes. $N$ Engl J Med 2015;373:2117-28.

143. Szalat A, Perlman A, Muszkat M, et al. Can SGLT2 inhibitors cause acute renal failure? plausible role for altered Glomerular Hemodynamics and Medullary Hypoxia. Drug Saf 2018;41:239-52.

144. Hahn K, Ejaz AA, Kanbay M, et al. Acute kidney injury from SGLT2 inhibitors: potential mechanisms. Nat Rev Nephrol 2016;12:711-2.

145. Nadkarni GN, Ferrandino R, Chang A, et al. Acute Kidney Injury in Patients on SGLT2 inhibitors: a propensity-matched analysis. Diabetes Care 2017:40:1479-85.

146. Ogawa W, Sakaguchi K. Euglycemic diabetic ketoacidosis induced by SGLT2 inhibitors: possible mechanism and contributing factors. J Diabetes Investig 2016;7:135-8.

147. Jensen ML, Persson F, Andersen GS, et al. Incidence of Ketoacidosis in the Danish Type 2 Diabetes Population Before and After Introduction of Sodium-Glucose Cotransporter 2 Inhibitors-A Nationwide, Retrospective Cohort Study, 1995-2014. Diabetes Care 2017:40:e57-8. 
148. Fralick M, Schneeweiss S, Patorno E. Risk of Diabetic Ketoacidosis after Initiation of an SGLT2 Inhibitor. N Engl J Med 2017;376:2300-2.

149. Fadini GP, Bonora BM, Avogaro A. SGLT2 inhibitors and diabetic ketoacidosis: data from the FDA Adverse Event Reporting System. Diabetologia 2017;60:1385-9.

150. lijima $\mathrm{H}$, Kifuji $\mathrm{T}$, Maruyama $\mathrm{N}$, et al. Pharmacokinetics, Pharmacodynamics, and Safety of Canagliflozin in Japanese Patients with Type 2 Diabetes Mellitus. Adv Ther 2015;32:768-82.

151. Devineni D, Polidori D. Clinical Pharmacokinetic, Pharmacodynamic, and Drug-Drug Interaction Profile of Canagliflozin, a Sodium-Glucose Co-transporter 2 Inhibitor. Clin Pharmacokinet 2015:54:1027-41.

152. Devineni D, Polidori D, Curtin CR, et al. Pharmacokinetics and pharmacodynamics of once- and twice-daily multiple-doses of canagliflozin, a selective inhibitor of sodium glucose co- transporter 2, in healthy participants. Int J Clin Pharmacol Ther 2015;53:438-46.

153. Devineni D, Curtin CR, Polidori D, et al. Pharmacokinetics and pharmacodynamics of canagliflozin, a sodium glucose cotransporter 2 inhibitor, in subjects with type 2 diabetes mellitus. $J$ Clin Pharmacol 2013;53:601-10.

154. Parkinson J, Tang W, Johansson CC, et al. Comparison of the exposure-response relationship of dapagliflozin in adult and paediatric patients with type 2 diabetes mellitus. Diabetes Obes Metab 2016;18:685-92.

155. Taylor SI, Blau JE, Rother KI. SGLT2-inhibitors trigger downstream mechanisms that may exert adverse effects upon bone. Lancet Diabetes Endocrinol 2015;3:8-10.

156. Loke YK, Price D, Herxheimer A. Systematic reviews of adverse effects: framework for a structured approach. BMC Med Res Methodol 2007;7:32 